\title{
Hamiltonian Formulation of Two-Dimensional Gyroviscous MHD
}

\author{
Philip J. Morrison*, I. L. Caldas **, and H. Tasso \\ Max-Planck-Institut für Plasmaphysik, EURATOM Association, Garching
}

Z. Naturforsch. 39a, 1023-1027 (1984); received August 9, 1984

\begin{abstract}
Gyroviscous MHD in two dimensions is shown to be a Hamiltonian field theory in terms of a non-canonical Poisson bracket. This bracket is of the Lie-Poisson type, but possesses an unfamiliar inner Lie algebra. Analysis of this algebra motivates a transformation that enables a Clebsch-like potential decomposition that makes Lagrangian and canonical Hamiltonian formulations possible.
\end{abstract}

\section{Introduction}

This work is concerned with the Hamiltonian field formulation of the equations that describe two-dimensional non-dissipative gyroviscous onefluid plasmas [1, 2, 3, 4]. These equations differ from the usual Eulerian form of ideal MHD by the inclusion of a non-entropy-producing stress tensor that arises from the finiteness of the ion gyroradius. The physics of this stress tensor is important for modelling tokamak discharges [5], and may be important for calculation of the MHD $\boldsymbol{k}$-spectrum by means of the partition function [6,7].

The Eulerian MHD equations in terms of their usual variables do not possess the form of a conventional Hamiltonian field-theory. Nonetheless, these equations were shown to be Hamiltonian in a generalized sense by incorporating a generalization of the Poisson bracket [8]. Generalized or noncanonical Poisson brackets possess the same algebraic properties as ordinary Poisson brackets, but the notion of canonical variables is abandoned and degeneracy is allowed. At present, noncanonical Poisson brackets for all of the major non-dissipative plasma systems have been obtained. For review of the formalism and applications the reader is directed to [9]-[12].

This paper is organized as follows: in Sect. II the two-dimensional gyroviscous MHD equations are described. In Sect. III we briefly review some aspects of the noncanonical Hamiltonian formalism

\footnotetext{
* Permanent address: Department of Physics and Institute for Fusion Studies, University of Texas at Austin, Austin, Texas 78712, USA. On leave June-August 1984. ** Permanent address: Instituto de Física, Universidade de São Paulo, C.P. 20516, 05508 São Paulo, S.P., Brazil. Partly supported by Fundação de Amparo à Pesquisa do Estado de São Paulo (FAPESP).
}

and then present the Poisson bracket for the gyroMHD model. Section IV is concerned with the algebraic interpretation of the bracket presented in Sect. III. In case the methods of this section are unfamiliar to the reader, it may be skipped without loss of continuity. Section V deals with Clebsch [13] potentials, i.e. a potential decomposition that transforms the noncanonical formalism into canonical form with gauge conditions. We thus obtain a variational principle. Clebsch-potentials are discussed in [9], [12], [14]-[18]. The Clebsch-potential decomposition presented here differs in form from all previous work. We conclude with Sect. VI.

\section{Equations of Motion}

The gyroviscous stress tensor is associated with a momentum flux that is due to Larmor gyration. Evidently, this effect occurs perpendicular to the magnetic field. Here we assume that the magnetic field has the form $\boldsymbol{B}=\boldsymbol{B} \boldsymbol{e}_{z}$, where $\boldsymbol{e}_{z}$ is a constant unit vector and $B$ is a scalar function that depends spatially only upon the cartesian coordinates ( $x$ and $y$ ) of the plane defined by $\boldsymbol{e}_{z}$. This same spatial dependence is assumed for the remaining dynamical variables: $\boldsymbol{M}, \varrho$, and $\beta$. Here $\boldsymbol{M}$ is the momentum density with components in the $x$ and $y$ directions given by $M_{s}(s=1,2)$. We assume $\boldsymbol{e}_{\boldsymbol{z}} \cdot \boldsymbol{M}=0$. The symbol $\varrho$ takes its usual meaning as the mass density, while $\beta \equiv P / B \mid$ where $P$ is the pressure. With these assumptions we obtain

$$
\begin{aligned}
\dot{M}_{s} & =-\partial_{i}\left(M_{i} M_{s} / \varrho\right)-\partial_{s}\left(\beta|B|+B^{2} / 2\right)-\partial_{i} \pi_{i s}, \\
\dot{Q} & =-\partial_{s} M_{s}, \\
\dot{B} & =-\partial_{s}\left(B M_{s} / \varrho\right), \\
\dot{\beta} & =-\partial_{s}\left(\beta M_{s} / \varrho\right),
\end{aligned}
$$


where the gyroviscous tensor $\pi_{i s}$ is given by

$$
\pi_{i s}=N_{s j i k} \beta \partial_{k}\left(M_{j} / Q\right)
$$

and

$$
N_{s j i k}=c\left(\delta_{s k} \varepsilon_{j i}-\delta_{j i} \varepsilon_{s k}\right) .
$$

Here $c$ is a dimensional constant, $\delta_{s k}$ is the Kronecker delta and $\varepsilon_{j i}$ is the antisymmetric tensor with components

$$
\varepsilon_{i j}=\left\{\begin{array}{rcc}
1 & \text { for } i=1, & j=2, \\
-1 & \text { for } i=2, & j=1, \\
0 & \text { for } i=j . &
\end{array}\right.
$$

We remark that in spite of the presence of the $\pi_{i s}$, Eqs. (1)-(4) conserve energy, momentum, angular momentum and center of mass motion given respectively by

$$
\begin{aligned}
H & =\int\left(M^{2} / 2 \varrho+\beta B+B^{2} / 2\right) \mathrm{d} \tau, \\
\boldsymbol{P} & =\int \boldsymbol{M} \mathrm{d} \tau, \\
\boldsymbol{L} & =\int \boldsymbol{x} \times \boldsymbol{M} \mathrm{d} \tau, \\
\boldsymbol{G} & =\int(\varrho \boldsymbol{x}-t \boldsymbol{M}) \mathrm{d} \tau,
\end{aligned}
$$

where $\mathrm{d} \tau=\mathrm{d} x \mathrm{~d} y$. In Sect. III we will discuss additional constants known as Casimirs.

\section{Noncanonical Hamiltonian Description}

The conventional version of Hamiltons equations for fields can be written in the form

$$
\dot{\psi}^{k}=\left\{\psi^{k}, H\right\}, \quad k=1,2, \ldots, 2 N,
$$

where $H$ is the Hamiltonian functional, $\psi^{k}$ denotes the field variables and the Poisson bracket $\{$,$\} of$ two functionals $F$ and $G$ is given by

$$
\{F, G\}=\int \frac{\delta F}{\delta \psi^{k}} O^{k l} \frac{\delta G}{\delta \psi^{l}} \mathrm{~d} \tau .
$$

The quantity $O^{k l}$ is given by

$$
\left(O^{k \prime}\right)=\left(\begin{array}{cc}
0 & I_{N} \\
-I_{N} & 0
\end{array}\right)
$$

where $I_{N}$ is the $N \times N$ unit matrix and the symbol $\delta F / \delta \psi^{k}$ means the usual functional derivative defined by

$$
\frac{\mathrm{d}}{\mathrm{d} \varepsilon} F\left[\psi^{k}+\varepsilon \delta \psi\right]_{\varepsilon=0}=\int \frac{\delta F}{\delta \psi^{k}} \delta \psi \mathrm{d} \tau .
$$

The bracket [Eq. (13)] assigns the new functional $\{F, G\}$ to the two given ones $F$ and $G$, and has the following important properties:

(i) $\{F, G\}$ is linear in $F$ and $G$,

(ii) $\{F, G\}=-\{G, F\}$,

(iii) $\{E,\{F, G\}\}+\{F,\{G, E\}\}+\{G,\{E, F\}\}=0$,

(iv) $\{E F, G\}=E\{F, G\}+\{E, G\} F$.

Because of properties (i) - (iii) the product defined by Eq. (13) acting on the vector space of functionals defines a Lie algebra. We note the (ii) and (iii) depend upon the form of $O^{k l}$, while $O^{k l}$ need only be independent of $F$ and $G$ for properties (i) and (iv).

The noncanonical generalization of Hamilton's formulation stresses the properties (i)-(iv) rather than requiring the specific form $O^{k l}$ given by (14). Indeed this is a generalization since fields with an odd number of components can now be put into the form of (12). In general, $O^{k l}$ can be a matrix operator that may, for example, be differential as well as have dependence on the $\psi^{k}$.

The bracket for gyro-MHD has two parts: the first is essentially that given for ideal MHD in [8], while the second (new) part contains higher derivatives (due to dispersion introduced by $\pi_{i s}$ ). The total bracket is given by

$$
\begin{aligned}
&\left\{F, G_{j}^{\prime}=-\int\left[M_{l}\left(\frac{\delta F}{\delta M_{k}} \partial_{k} \frac{\delta G}{\delta M_{l}}-\frac{\delta G}{\delta M_{k}} \partial_{k} \frac{\delta F}{\delta M_{l}}\right)\right.\right. \\
&+\varrho\left(\frac{\delta F}{\delta M_{k}} \partial_{k} \frac{\delta G}{\delta Q}-\frac{\delta G}{\delta M_{k}} \partial_{k} \frac{\delta F}{\delta Q}\right) \\
&+B\left(\frac{\delta F}{\delta M_{k}} \partial_{k} \frac{\delta G}{\delta B}-\frac{\delta G}{\delta M_{k}} \partial_{k} \frac{\delta F}{\delta B}\right) \\
&+\beta\left(\frac{\delta F}{\delta M_{k}} \partial_{k} \frac{\delta G}{\delta \beta}-\frac{\delta G}{\delta M_{k}} \partial_{k} \frac{\delta F}{\delta \beta}\right. \\
&\left.\left.-N_{i j s t}\left[\partial_{s} \frac{\delta F}{\delta M_{i}}\right]\left[\partial_{t} \frac{\delta G}{\delta M_{j}}\right]\right)\right] \mathrm{d} \tau .
\end{aligned}
$$

It is not difficult to see that Eqs. (1)-(4) are concisely given by

$$
\begin{aligned}
\dot{M}_{s} & =\left\{M_{s}, H\right\}, & \dot{\varrho} & =\{\varrho, H\}, \\
\dot{B} & =\{B, H\}, & \dot{\beta} & =\{\beta, H\},
\end{aligned}
$$

where $H$ is the energy, see (8). 
Equation (16) can be seen to satisfy property (ii) by integration by parts and neglect of surface terms. Also by the same procedure the operator $O^{k l}$ can be extracted - we do not do this here since the form of (16) as it stands is more transparent for reasons that will be discussed in Sect. IV, where the Jacobi identity [property (iii)] is shown.

Before closing this section we point out that associated with noncanonical Poisson brackets are special constants of motion known as Casimirs. These are bracket constants; i.e. constants that do not depend upon the specific form of the Hamiltonian. They are associated with the degeneracy of the bracket; if $C$ is a Casimir then $\{C, F\}=0$ for all functionals $F$. Casimirs for (16) are given by

$$
C=\sum_{i} \lambda_{i} \int Q^{a_{i}} B^{b_{i}} \beta^{c_{i}} \mathrm{~d} \tau
$$

provided $a_{i}+b_{i}+c_{i}=1$ for all $i$. Here the $\lambda_{i}$ 's are arbitrary constants. More generally it is not difficult to show that the following commutes with all functionals

$$
C=\int \varrho f\left(\frac{B}{\varrho}, \frac{\beta}{\varrho}\right) \mathrm{d} \tau,
$$

where $f$ is an arbitrary function. We note that in (18) $\varrho, B$ and $\beta$ can be permuted. These Casimirs are independent of the gyro term.

\section{Lie-Poisson Interpretation}

The natural situation for quantum field theories, such as the Klein-Gordon or the vacuum Maxwell equations, is that the operator $O^{k l}$ does not have explicit dependence upon the field variables. The standard form of $O^{k l}$ for these fields is the canonical case. This is not the typical situation for Eulerian field equations that describe continuous media. For example, in the case of the ideal fluid, ideal MHD or the Vlasov-Poisson equations, the natural form of the operator $O^{k l}$ has linear dependence upon the field variables. Specifically, Poisson brackets for media have the form

$$
\{F, G\}=\int \psi^{i}\left[\frac{\delta F}{\delta \psi}, \frac{\delta G}{\delta \boldsymbol{\psi}}\right]_{i} \mathrm{~d} \tau,
$$

where $[,]_{i}$ is the $i^{\text {th }}$ component of the product for a Lie algebra of vector-valued functions. Brackets of this type have recently been termed Lie-Poisson. (See e.g. [10], [17], and [11].)
From the form of (19) it is clear that there are two Lie algebras involved: an inner Lie algebra where the vector space is composed of vectorvalued functions and an outer Lie algebra where the vector space is composed of functionals. When this situation occurs the Jacobi identity for the outer algebra is a consequence of that for the inner algebra. (This can be shown directly by use of the techniques discussed in [9].) Thus we see the importance of studying the inner algebra. Additionally, brackets of the form of (19) can be classified by their inner algebra; hence, field equations that have brackets of this form can also be classified.

We denote the inner Lie algebra of gyro-MHD by $G_{5}$. Any element $f$ of $G_{5}$ can be written as

$$
\boldsymbol{f}=\left(f_{1}, f_{2}, f_{\varrho}, f_{B}, f_{\beta}\right),
$$

where $f_{1}, f_{2}, f_{\varrho}, f_{B}$, and $f_{\beta}$ are sufficiently differentiable functions defined on a domain, which has coordinates $x$ and $y$. The product of two elements $f$ and $\boldsymbol{g}$ in $G_{5}$ is given by

$$
\begin{aligned}
{[\boldsymbol{f}, \boldsymbol{g}]=} & \left(f_{i} \partial_{i} g_{1}-g_{i} \partial_{i} f_{1}, f_{i} \partial_{i} g_{2}-g_{i} \partial_{i} f_{2},\right. \\
& f_{i} \partial_{i} g_{\varrho}-g_{i} \partial_{i} f_{\varrho}, f_{i} \partial_{i} g_{B}-g_{i} \partial_{i} f_{B}, f_{i} \partial_{i} g_{\beta} \\
& -g_{i} \partial_{i} f_{\beta}-a_{s j}\left(\partial_{s} g_{i}\right)\left(\partial_{i} f_{j}\right) \\
& \left.+a_{s j}\left(\partial_{i} g_{j}\right)\left(\partial_{s} f_{i}\right)\right),
\end{aligned}
$$

where repeated indicies are to be summed only on 1 and 2 and the quantities $a_{s j}$ are real constants that for now need not be further specified.

The first two components of the product given by Eq. (20) have the familiar form of the Lie algebra that corresponds to diffeomorphisms of our domain, which for purposes here we can take to be the plane. If we set the $a_{s j}$ to zero, then our algebra corresponds to a semi-direct product extension of the diffeomorphism algebra. This sort of inner algebra is basic to ideal and reduced MHD [16, 10, 17, 11]. Here the addition of the $a_{s j}$ alters this form by including a higher degree of differentiation in the last component. Nevertheless, it is straight forward to show directly that (20) satisfies the Jacobi identity for all constants $a_{s j}$. This is unnecessary as we will subsequently show.

As in the case of the semi-direct product algebras, elements of the form $\left(0,0, f_{\varrho}, f_{B}, f_{\beta}\right)$ constitute an ideal of $G_{5}$ (with null second power); but $G_{5}$ does not possess a subalgebra with elements of the form $\left(f_{1}, f_{2}, 0,0,0\right)$. By examination of $(20)$ we ob- 
serve that $G_{5}$ does possess a subalgebra with elements of the form

$$
\boldsymbol{f}=\left(f_{1}, f_{2}, 0,0, a_{s j} \partial_{s} f_{j}\right)
$$

Equation (21) suggests a transformation $i$ such that

$$
i:\left(f_{1}, f_{2}, f_{\varrho}, f_{B}, f_{\beta}\right) \rightarrow\left(f_{1}, f_{2}, f_{\varrho}, f_{B}, f_{\beta}+a_{s j} \partial_{s} f_{j}\right) \text {. }
$$

This transformation is 1 to 1 and linear. We observe that

$$
i^{-1}[i(\boldsymbol{f}), i(\boldsymbol{g})]=[\boldsymbol{f}, \boldsymbol{g}]_{\mathrm{SDP}},
$$

where $[\boldsymbol{f}, \boldsymbol{g}]_{\mathrm{SDP}}$ is the semi-direct product product. Thus by this isomorphism we see that $G_{5}$ must satisfy the Jacobi condition for all $a_{s j}$. If we pick $a_{s j}=c \varepsilon_{s j}$ then $G_{5}$ is seen to be the inner algebra for the bracket of (16); hence, the gyro-bracket must satisfy the Jacobi identity. The physical interpretation of $i$ will be discussed in Section VI.

\section{Variational Principle - Clebsch Representation}

In order to obtain a variational principle and a canonical Hamiltonian description for (1)-(4), a representation for $\boldsymbol{M}$ in terms of potentials must be introduced. Motivated by the transformation $i$ of Sect. IV we express $\boldsymbol{M}$ as follows:

$$
M_{s}=\varrho \partial_{s} \chi+B \partial_{s} \psi+\beta \partial_{s} \alpha-c \varepsilon_{s k} \partial_{k} \beta,
$$

where the quantities $\chi, \psi$ and $\alpha$ are Clebsch-like potentials. We note that the canonical description requires the introduction of "gauge" conditions; i.e. the physical state $\boldsymbol{M}$ does not uniquely determine the potentials on the RHS of (22). Unlike the connection in electrodynamics between the magnetic field and the vector potential, (22) has quadratic dependence upon the field variables. This situation arises because Poisson brackets for media are naturally Lie-Poisson.

Equations (1) - (4) can be shown to be implied by the Euler-Lagrange equations obtained from the variational principle

$$
\delta \iint \mathcal{d} \tau \mathrm{d} t=0,
$$

where the Lagrangian density is given by

$$
\begin{aligned}
f= & -M^{2} / 2 Q-\beta B-B^{2} / 2 \\
& -(\beta \dot{x}+B \dot{\psi}+Q \dot{\psi}) .
\end{aligned}
$$

Here $M$ is a shorthand for the expression given by (22). $f$ can be shown to be numerically equal to the total pressure $B^{2} / 2+P$.
Evidently $f$ is a first order Lagrangian of the form $p \dot{q}-H$; hence we define:

$$
\pi_{\%} \equiv-Q, \quad \pi_{\psi} \equiv-B \quad \text { and } \quad \pi_{\alpha} \equiv-\beta .
$$

The Euler-Lagrange equations of Eqs. (23) can thus be written in the following form:

$$
\begin{array}{ll}
\dot{\psi}=\{\chi, H\}_{c}, & \pi_{\chi}=\left\{\pi_{\chi}, H\right\}_{c}, \\
\dot{\psi}=\{\psi, H\}_{c}, & \pi_{\psi}=\left\{\pi_{\psi}, H\right\}_{c}, \\
\dot{\alpha}=\{\alpha, H\}_{c}, & \pi_{x}=\left\{\pi_{\alpha}, H\right\}_{c},
\end{array}
$$

where $H$ is given by Eq. (8) with the substitution of Eq. (22), and $\{,\}_{c}$ stands for the canonical bracket; i.e.

$$
\begin{gathered}
\{F, G\}_{c}=\int\left(\frac{\delta F}{\delta \chi} \frac{\delta G}{\delta \pi_{\%}}-\frac{\delta F}{\delta \pi_{\chi}} \frac{\delta G}{\delta \chi}+\frac{\delta F}{\delta \psi} \frac{\delta G}{\delta \pi_{\psi}}-\frac{\delta F}{\delta \pi_{\psi}} \frac{\delta G}{\delta \psi}\right. \\
\left.+\frac{\delta F}{\delta \chi} \frac{\delta G}{\delta \pi_{\chi}}-\frac{\delta F}{\delta \pi_{\chi}} \frac{\delta G}{\delta \chi}\right) \mathrm{d} \tau
\end{gathered}
$$

We note that the connection between the bracket $\{$,$\} and the noncanonical bracket of (16) can be$ made by assuming that the functionals $F$ and $G$ in (25) obtain their dependence upon $\chi, \pi_{\chi}$, etc. through $\boldsymbol{M}, \varrho$, etc. In this way, for example, functional derivatives with respect to $\chi$ can be $\boldsymbol{M}$. Thus (25) can be transformed to (16).

\section{Conclusions}

We have presented the noncanonical Poisson bracket for gyroviscous MHD in two dimensions, together with a class of Casimir invariants. This bracket is seen to be Lie-Poisson, a standard type for continuous media. The associated inner Lie algebra was presented and identified by making use of the transformation $i$ of Section IV. Using this transformation a variational principle and a canonical Hamiltonian description were obtained.

The transformation $i$ corresponds physically to a change of reference frames. If we transform the momentum density according to $M_{i}^{\prime}=M_{i}-c \varepsilon_{k i} \partial_{k} \beta$, then Eqs. (1) - (4) written in terms of $M_{i}^{\prime}$ have a Poisson bracket representation using the ideal MHD bracket together with the Hamiltonian

$$
\begin{aligned}
H=\int[ & M^{\prime 2} / 2 Q+\frac{c}{\varrho} \varepsilon_{k i} M_{i}^{\prime} \partial_{k} \beta+\frac{c^{2}}{2 \varrho}\left(\partial_{i} \beta\right)\left(\partial_{i} \beta\right) \\
& \left.+\beta B+B^{2} / 2\right] \mathrm{d} \tau .
\end{aligned}
$$


The relative velocity of the primed and unprimed frames is given by

$$
v=\frac{\boldsymbol{B} \times \nabla P}{n q B^{2}}-\frac{P \boldsymbol{B} \times \nabla|B|}{n q B^{3}} .
$$

The first term of (26) corresponds to the well-known diamagnetic drift velocity, while the second term is seen to be minus an average of the particulate $\nabla B$ drift velocity, since $P=m / 2\left\langle v_{\perp}^{2}\right\rangle$. By going to a frame moving at $v$ the Hamiltonian formalism takes a more natural form. We thus see that the Casimir constants for two dimensional gyro-MHD must be in a 1 to 1 correspondence with those for ideal MHD. Ramifications of this in three dimensions will be reported in the future [19].

One application of the model and formalism presented here is to calculate the $\boldsymbol{k}$-spectrum by integrating the partition function $[6,7]$. This is most clearly understood in terms of a Hamiltonian formalism. Although it is unlikely that the two-dimensional functional integral encountered here can be evaluated analytically, a Montecarlo type computer

[1] S. Chapman and T. Cowling, The Mathematical Theory of Non-Uniform Gases, Cambridge University Press, New York 1953.

[2] W. A. Newcomb, Dynamics of a Gyroviscous Plasma, in "Dynamics of Fluids and Plasmas", Edited by S. I. Pai et al., Academic Press, New York 1966.

[3] A. N. Kaufman, Phys. Fluids 3, 610 (1960).

[4] H. Tasso and P. P. J. M. Schram, Nuclear Fusion 6, 284 (1966).

[5] R. D. Hazeltine, M. Kotschenreuther, and P. J. Morrison (under preparation).

[6] H. Tasso and K. Lerbinger, Phys. Lett. 97 A, 384 (1983).

[7] I. L. Caldas and H. Tasso, Fluctuation Spectrum for Linear Gyroviscous MHD, Report IPP 6/237, D-8046 Garching b. München, Max-Planck-Institut für Plasmaphysik (1984). Physics Letters 104A, 423 (1984).

[8] P. J. Morrison and J. M. Greene, Phys. Rev. Lett. 45, 790 (1980); 48, 569 (1982).

[9] P. J. Morrison, Poisson Brackets for Fluids and Plasmas, in "Mathematical Methods in Hydrodynamics and Integrability in Related Dynamical Systems", AIP Conf. Proc., \# 88, La Jolla, Ca., 1982, M. Tabor and Y. Treve (Eds).

[10] J. E. Marsden, A. Weinstein, T. Ratiu, R. Schmid, and R. G. Spencer, Atti delle Academia della Scienze di Torino Suppl. 117, 289 (1983). calculation [20] appears possible. The gyro-model presented here is intriguing since knowledge [6] obtained from one-dimensional functional integration suggests that the dispersion introduced by gyroviscosity may be crucial for eliminating the ultraviolet divergence.

In closing we mention that the formalism presented here can be useful for stability analysis [21, 22]. Using the Casimir invariants, variational principles for equilibria can be constructed and in many cases criteria for nonlinear Liapunov stability have been found.

\section{Acknowledgements}

One of us (PJM) would like to thank J. E. Marsden for many useful conversations and also thank the IPP for their hospitality. His research was supported in part by DOE contracts DE-FG05-80ET53088 and DE-AT03-82ER-12097. Another of us (ILC) would also like to thank the IPP for their hospitality as well as the FAPESP for financial support.

[11] J. E. Marsden and P. J. Morrison, Contemporary Math. 28 (1984) (AMS Conf. Proc., Bouldar, CA).

[12] P. J. Morrison and R. D. Hazeltine, Physics of Fluids 27, 886 (1984).

[13] A. Clebsch, J. reine angew. Math. 56, 1 (1859).

[14] R. Seliger and G. Whitham, Proc. Roy. Soc. London A305, 1 (1968).

[15] V. Zakharov and E. Kuznetzov, Sov. Phys. Dokl. 15,913 (1971).

[16] D. Holm and B. Kupershmidt, Physica 6D, 347 (1983).

[17] J. Marsden, T. Ratiǔ, and A. Weinstein, "Semi-direct products and reduction in mechanics", Trans. Amer. Math. Soc. (to appear).

[18] I. L. Caldas and H. Tasso, Z. Naturforsch. 39a, 9 (1984).

[19] P. J. Morrison, I. L. Caldas, and H. Tasso (under preparation).

[20] N. Metropolis, A. Rosenbluth, M. Rosenbluth, A. Teller, and E. Teller, J. Chem. Phys. 21, 1087 (1953).

[21] D. D. Holm, J. E. Marsden, T. Ratiu, and A. Weinstein, Phys. Lett. 98 A, 15 (1983); also manuscript under preparation.

[22] R. D. Hazeltine, D. D. Holm, J. E. Marsden, and P. J. Morrison, ICPP Proc. Lausanne 1984, p. 204; also issued as Institute for Fusion Studies Report IFSR \# 139 (1984), Austin, Texas. 